\title{
New receivers for DS-SS in time variant multipath channels based on the PN alignment concept
}

\author{
J.C. Socoró, J.Ll. Pijoan, , J.A. Morán \\ Department of Signal Theory and Communications \\ La Salle School of Engineering -Ramon Llull \\ University (URL) \\ Pg. Bonanova 8, 08022 Barcelona, Spain \\ e-mail: jclaudi@salleURL.edu
}

\author{
F. Tarres \\ Polytechnic University of \\ Catalunya (UPC) \\ Campus Nord UPC - 08034 \\ Barcelona, Spain
}

\begin{abstract}
In this paper we present new combined blind equalization and detection schemes for a DSSS system. The new proposed algorithms improve the bit error rate compared to traditional RAKE receivers in time-variant channels with multipath. This improvement is obtained in both simulated and a real ionospheric HF link. Its very low computational complexity makes them suitable to be implemented in real receivers.
\end{abstract}

\section{INTRODUCTION}

It is well known that RAKE receivers benefit from time diversity in the received signal compared to single delay receiver [1] [6]. The combination of the different finger demodulated components reduces thus the bit error rate increasing the apparent signal to noise ratio. In this paper we present two new blind equalization and detection techniques for DS-SS systems based on the new concept of $P N$ alignment. They use a filter that attempts to align constructively all the delayed components in the output signal of a frequency selective and multipath channel. This is achieved by the definition of a suitable cost function that uses the known transmitted spreading code. Both algorithms use the steepest descent philosophy to update the filter coefficients and they have an very low computational complexity.

This work has been focused on a real ionospheric point to point link ${ }^{1}$ established between Huelva and Barcelona in Spain [7], where time variability, multipath components and low SN ratios (due to both noise and interferences) make difficult to achieve good bit error rates if error correcting techniques are not used. The use of direct sequence spread spectrum modulation is justified by its robustness to multipath and low SNR, and its high privacy of the communication. By the time of writing this article, those methods have only been tested with a single user.

First of all, a brief presentation of the HF link and the channel model is carried out in section II. After the algorithms explanation in section III, some computer simulation results are shown in section IV, as well as some real results obtained in the ionospheric link. In these results the new two schemes and a standard RAKE receiver [1] are compared together. Finally, in section $\mathrm{V}$ we emphasize the main conclusions of this work and future lines.

\section{DESCRIPTION OF THE LINK}

The main features of our ionospheric link are: it is a simplex point to point link of about $800 \mathrm{Km}$ distance between Huelva (the transmitter) and Barcelona (the receiver) with
$200 \mathrm{~W}$ of maximum transmission power, the carrier frequency is between $4-8 \mathrm{MHz}$, and DPSK is used as data modulation A fully digital transmitter and receiver with direct analog-todigital conversion at the HF band is implemented, and the receiver processes the baseband signal with 3 DSP's working in parallel.

This system has two operation modes: the channel evaluation mode, where a test signal is transmitted every minute 24 hours a day in order to get the Scattering function; the real-time operation mode, where information is transmitted and received in real-time. In this case, some acquisition, demodulation, equalization and detection schemes in low SNR environment with narrow-band interference, long channel impulse response and long fadings have to be accurately designed [6] [8]. A high speed acquisition and processing hardware has been designed to implement the functions above mentioned. The basic system configuration is shown in figure 1. There are four blocs (transmitter, receiver, channel evaluation and real-time system) that can be combined in order to work in the two operation modes. See [7] and [8] for more details.

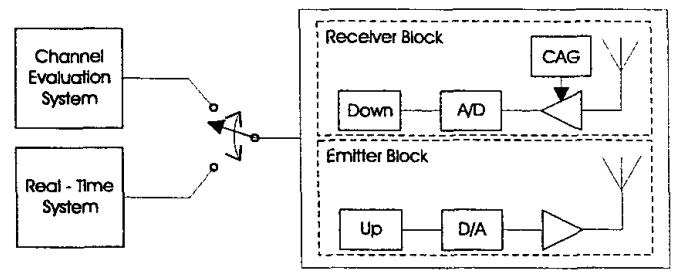

Fig. 1: Block diagram of the system's hardware

The ionosphere behaves as a time varying multipath channel, and it can be modeled [1] as a tapped delay line with time varying coefficients $\mathbf{h}_{l}=\left[h_{0}(l), h_{1}(l), \ldots, h_{N-1}(l)\right]^{T}$. When the receiver sampling is faster than the symbol rate $R_{s}$, it is also possible to use a multichannel model (commonly used in order to formulate fractionally spaced equalizers [2]). In this case, we can state that $h_{k}(l)=h\left(\tau=k / R_{s} ; t=l \cdot N / R_{s}\right)$, where $h(\tau ; t)$ is the analog baseband impulse response of the channel, and $N$ is the process gain. In this model the channel is supposed to remain almost constant during a symbol period. This is a very standard supposition, specially in HF communications where Doppler frequencies are about $1 \mathrm{~Hz}$ [4] [5] (both shift and spread phenomena). Another important hypothesis

${ }^{1}$ This work was supported by the research project CICYT TIC97-0787-C02-01 financed by the Spanish Government. 
is that the impulse response duration is lower than the symbol period, as only very low-rate communications are here used.

The binary sequence $\mathrm{I}_{l}$ is the binary information sequence $S_{l}$ differentially encoded. The $\mathrm{N}$-chip pseudonoise sequence (PN) or spreading code is $\mathbf{p}=\left[p_{0}, p_{1}, \ldots, p_{N-1}\right]^{T}$. The spreading process can be shown as an $N$ expansion followed by a FIR filter with impulse response $p$, as it is shown in the serial model of figure 2. After the spreading and the time-variant linear distortion, white Gaussian complex noise $n_{n}$ of power $\sigma^{2}$ is added.

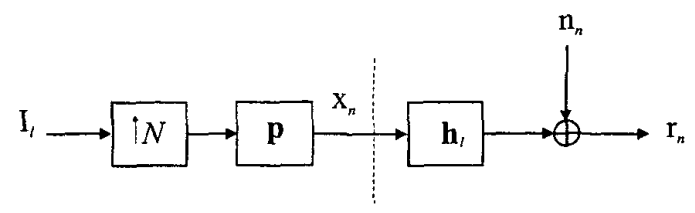

Fig. 2: Serial discrete model for the spreading procedure at the transmitter and the linear channel. The taps of vector $\mathbf{h}_{l}$ are time variant

On the other hand, in the multichannel model shown in figure 3 , the channel is viewed as $N$ parallel subchannels with time-variant impulse responses $\mathbf{g}_{k}(l)=\left[g_{k}^{0}(l), g_{k}^{1}(l)\right]^{T}$, where: $\quad g_{k}^{i}(l) \hat{=} p h_{(k+i N)}(l)$ for $k=1,2, \ldots, N$ and $i=$ 0,1 ; the combined spreading filter and channel vector is $\mathbf{p h},=\left[p h_{0}(l), p h_{1}(l), \ldots, p h_{2 N-2}(l)\right]^{T}=\mathbf{p} * \mathbf{h}_{l}$, and $*$ is the convolution operator. Every noise sequence $\mathbf{n}_{k}(l)$ of each $k$-subchannel is related with the original noise with $\mathbf{n}_{k}(l) \hat{=} \mathbf{n}_{(k+l N)}$. Then the output of the multichannel model is a vector of length $N$ that changes every transmitted symbol $\mathrm{I}_{l}$.

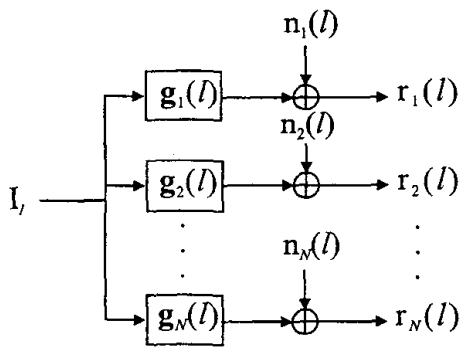

Fig. 3: Multichannel discrete model for the spreading procedure at the transmitter and the linear channel.

\section{NEW PN ALIGNMENT-BASED ALGORITHMS}

As it has been commented before, the new proposed schemes are based on a filter (or equalizer) that combines all the useful PN sequence energy in the received signal to perform a reconstruction by means of the alignment of all these delayed components. The two proposed techniques are: i) a blind matrix-based alignment filter and, ii) a blind multichannel-based alignment filter.

BLIND MATRIX-BASED PN ALIGNMENT FILTER
In this subsection the first proposed algorithm is presented. It uses a matrix filtering principle, in order to obtain an output vector that combines the better signal energy components of the incoming signal. The algorithm update equation and the detection process work every $N$ input samples. The filter update procedure searches the maximum of a cost function $\mathbf{J}_{M}$ with the constraint of constant energy at the output in order to avoid the divergence of the method.

First of all, a bloc diagram of the proposed algorithm is shown in figure 4.

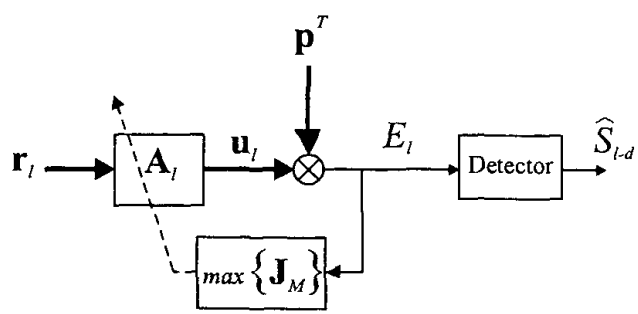

Fig. 4: Blind matrix-based filter for PN alignment.

The received signal $r_{n}$ from serial model of figure 2 feeds a shift register $\mathbf{r}_{l}$ of $R$ positions $(R \geq N$ ). This register is updated every $N$ input samples and thus the signal vector $\mathbf{r}_{l}$ is defined as: $\mathbf{r}_{l}=\left[r_{(l N)}, r_{(l N-1)}, \ldots, \boldsymbol{r}_{(l N-R+1)}\right]^{T}$ where $l \in Z$ is the transmitted symbol index. The equalizer uses a mix matrix $\mathbf{A}_{l}$ of dimensions $N \times R$ and complex coefficients, this is: $A_{l}=\left(a_{i j}^{l}\right)_{1 \leq i \leq N, 1 \leq j \leq R}$ and $a_{i j}^{l} \in C$. The time index $l$ show the adaptive nature of this matrix. The update of this matrix searches for the maximum of a cost function $\mathbf{J}_{M}$ following the gradient direction:

$$
\mathbf{A}_{l+1}=\mathbf{A}_{l}+\mu \frac{\partial \mathbf{J}_{M}}{\partial \mathbf{A}^{*}}
$$

where $\mu$ is a step size parameter that controls the convergence properties of the algorithm.

The output vector $\mathbf{u}_{l}$ of dimension $N \times 1$ has the input information of $\mathbf{r}_{l}$ mixed with the mix matrix as:

$$
\mathbf{u}_{l}=\mathbf{A}_{l} \mathbf{r}_{l}
$$

The equalizer has the purpose of detecting all the multipath components of the transmitted signal in $\mathbf{r}_{\text {! }}$ (with the same signature $p$ ) and to recombine them together to enhance the SNR before the final detection (like a RAKE receiver does) Then, the vector $\mathbf{u}_{l}$ is named the aligned vector, because the equalizer take all these multipath signals and it performs a constructive addition with the same time position and the same phase. The result must be the PN sequence multiplied by the information $\mathrm{I}_{t-d}$ (where $d$ is detection delay between 0 and 1) and by a slow time varying complex coefficient (due to the channel dynamic behavior).

Before the detection, the aligned vector is multiplied by the PN sequence $\mathbf{p}$ and accumulated:

$$
E_{l}=\mathbf{p}^{T} \mathbf{u}_{l}
$$

The cost function is defined as the energy of the demodulated sample $E_{l}$, in order to denote the desired high instantaneous cross-correlation between the aligned vector and the PN sequence:

$$
\mathbf{J}_{1} \triangleq\left|E_{l}\right|^{2}
$$


Substituting the equations (2) and (3) in (4), we obtain:

$$
\mathbf{J}_{1}=\mathbf{p}^{\boldsymbol{T}} \mathbf{A}_{l} \mathbf{r}_{l} \mathbf{r}_{l}^{H} \mathbf{A}_{l}^{H} \mathbf{p}
$$

If $\mathbf{J}_{1}$ is maximized with respect to the mix matrix $\mathbf{A}_{l}$, the trivial solution $a_{i j} \rightarrow \infty$ is found. To avoid this, it is necessary to force a constraint $\mathbf{W}$ like (i.e.) making the aligned vector of constant module, and then a stable solution to the problem is found. So the final cost function includes the restriction with its corresponding Lagrange multiplier $\lambda$ :

$$
\begin{aligned}
& \mathbf{J}_{M}=\mathbf{J}_{1}+\lambda \mathbf{W}=\left|E_{l}\right|^{2}+\lambda\left(\left\|\mathbf{u}_{l}\right\|_{2}^{2}-1\right) \\
& =\mathbf{p}^{T} \mathbf{A}_{l} \mathbf{r}_{l} \mathbf{r}_{l}^{H} \mathbf{A}_{l}^{H} \mathbf{p}+\lambda\left(\mathbf{r}_{l}^{H} \mathbf{A}_{l}^{H} \mathbf{A}_{l} \mathbf{r}_{l}-1\right)
\end{aligned}
$$

We can see that $\mathbf{J}_{M}$ is a quadratic function of mix matrix coefficients $a_{i j}$ and it has a single maximum, so a steepest ascent approach can be used to reach this maximum.

The next step is to maximize (6) with respect to $\mathbf{A}_{l}$ and to isolate the optimmum value of the Lagrange multiplier setting the result to zero vector. After substituting the optimum value $\lambda=-\left|E_{i}\right|^{2}$ the next gradient vector is found:

$$
\frac{\partial \mathbf{J}_{M}}{\partial \mathbf{A}^{*}}=E_{l}\left(\mathbf{p}-E_{l}^{*} \mathbf{u}_{l}\right) \mathbf{r}_{l}^{H}
$$

Finally, the last expression is substituted in (1) to give the filter update equation. The detection process after the equalizer/demodulator uses the demodulated sample $E_{l}$ to differentially decode each received bit as in [1]. The complete procedure of the proposed algorithm for both equalizing and detecting the received signal is summarized in the next table:

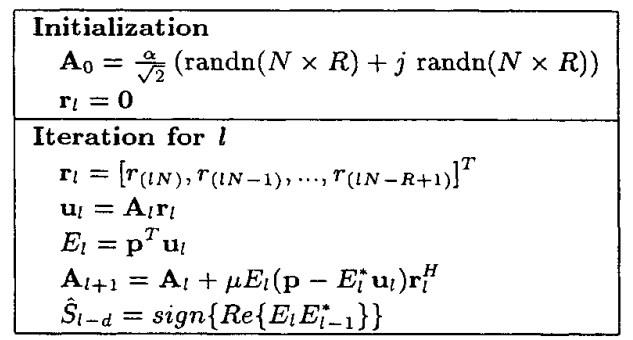

Tab. 1: Summary of the blind matrix-based PN alignment filter. Filter coefficients are initialized to zero mean complex Gaussian random numbers with variance $\alpha^{2}$.

This simple algorithm has a computational complexity of order $O(R)$ per input sample. In order to benefit from time diversity $R$ must be set to a value around $2 N$ or $3 N$, as it is supposed that the channel spreads a single repetition of $\mathbf{p}$ up to $2 N-1$ samples, and an asynchronous reception is used.

Performance of this method has a strong dependence on the election of the step size parameter $\mu$. There is a high sensibility (that can lead to a unstable situation) on both SNR and the speed of variation of the channel. After some simulations and real transmissions (see section IV), we have seen that a good choice is a variable step size like:

$$
\mu \hat{\hat{=}} \mu_{l}=\frac{\delta}{\left\|\mathbf{u}_{l}\right\|_{2}}
$$

Where $\|\bullet\|_{2}$ is the vector 2-norm and the parameter $\delta$ has to be dimensioned according to the channel.

This method is directly simplified if the total filter vector $\mathbf{b}_{l}^{H}=\mathbf{p}^{T} \mathbf{A}_{l}$ is defined. Then, a equivalent recursion is found:

$$
\begin{array}{r}
\mathbf{b}_{l+1}=\mathbf{b}_{l}+\mu E_{l}^{*}\left(N-\left|E_{l}\right|^{2}\right) \mathbf{r}_{l} \\
E_{l}=\mathbf{b}_{l}^{H} \mathbf{r}_{l}
\end{array}
$$

\section{BLIND MULTICHANNEL-BASED ALIGNMEǸT FILTER}

In this subsection we present an alternative solution to matrixbased equalizer. In fact, it is based exactly on the same principle, but it is formulated from the multichannel point of view. As it is shown later, similar or even better results are obtained with a considerably great reduction of computational complexity. The bloc diagram of this second method can be seen in figure 5 .

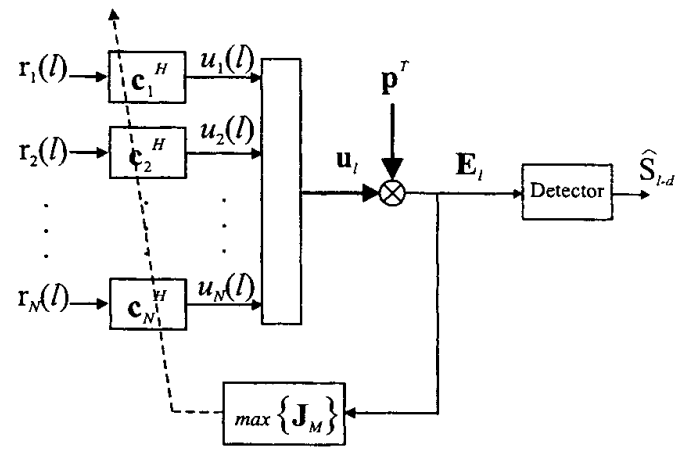

Fig. 5: Bloc diagram of the blind multichannel-based alignment filter.

This filter consists on $N$ parallel FIR filters of length $F$ and impulse responses $\mathbf{c}_{k}^{H}=\left[c_{1}^{k}, c_{2}^{k}, \ldots, c_{F}^{k}\right]$, and operates individually on each sample stream of the received vector in the multichannel model of figure 3

In this case, the aligned vector $\mathbf{u}_{l}$ is expressed by:

$\mathbf{u}_{l}=\left[u_{1}(l), u_{2}(l), \ldots, u_{N}(l)\right]^{T}=\left[\mathbf{c}_{1}^{H} \mathbf{r}_{1}(l), \mathbf{c}_{2}^{H} \mathbf{r}_{2}(l), \ldots, \mathbf{c}_{N}^{H} \mathbf{r}_{N}(l)\right]^{T}$

where $\mathbf{r}_{k}(l) \hat{=}\left[r_{k}(l), r_{k}(l-1), \ldots, r_{k}(l-F+1)\right]^{T}$ are the $F$ last output samples of the $\mathrm{k}$ th subchannel recorded in the $\mathrm{k}$ th fil ter.

The demodulated sequence $E_{l}$ of equation (3) can be expressed in function of $\mathrm{c}_{k}$ vectors as:

$$
E_{l}=\mathbf{p}^{T} \mathbf{u}_{l}=\sum_{k=1}^{N} p_{k} u_{k}(l)=\sum_{k=1}^{N} p_{k} \mathbf{c}_{k}^{H} \mathbf{r}_{k}(l)
$$

The cost function is now defined with the same criterion used in the previous subsection. Using equations (10) and (11) we can write:

$$
\begin{aligned}
& \mathbf{J}_{M}=E_{l} E_{l}^{*}+\lambda\left(\mathbf{u}_{l}^{H} \mathbf{u}_{l}-1\right)= \\
& \sum_{k=1}^{N} \sum_{i=1}^{N} p_{i}^{*} p_{k} \mathbf{r}_{i}^{H}(l) \mathbf{c}_{i} \mathbf{c}_{k}^{H} \mathbf{r}_{k}(l)+ \\
& +\lambda\left(\sum_{k=1}^{N} \mathbf{c}_{k}^{H} \mathbf{r}_{k}(l) \mathbf{r}_{k}^{H}(l) \mathbf{c}_{k}-1\right)
\end{aligned}
$$


The optimum solution is achieved when $\boldsymbol{J}_{M}$ reaches its maximum (when vector $\mathbf{u}_{l}$ is aligned with vector $\mathbf{p}$ ) or its gradients with respect to coefficient vectors $\mathbf{c}_{k}^{*}$ are zero. In order to use a steepest ascent approach, the same previous optimum Lagrange multiplier is found and substituted in the gradient vector equation. Finally, the update equation for the filter coefficient vector $\mathbf{c}_{k}$ is:

$$
\mathbf{c}_{k}(l+1)=\mathbf{c}_{k}(l)+\mu E_{l}^{*}\left(p_{k}-E_{l} u_{k}^{*}(l)\right) \mathbf{r}_{k}(l)
$$

where $c_{k}(l)$ is the coefficient vector of the $\mathrm{k}$ th filter at 1 th iteration.

We have found that the step size parameter $\mu$ plays a less critical role than in the first scheme, avoiding unstability without the need of a time variant step size parameter. The complete equalization and detection algorithm is summarized in table 2 .

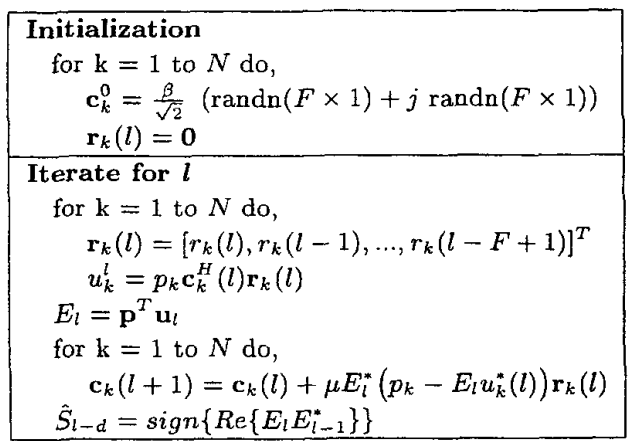

Tab. 2: Algorithm resume of the blind multichannel alignment filter. Filter coefficients are initialized to zero mean Gaussian random numbers with variance $\beta^{2}$.

The computational complexity of this second algorithm is of order $O(F)$ complex operations per input sample. It is very important to emphasize that this algorithm has a extremely good behavior for values of $F=1,2$ or 3 , in the type of analyzed HF channels, making it specially attractive for its implementation in real receivers.

Similarly to the first proposed method, the $\mathbf{p}$ dependence of the filtering procedure can be eliminated if vectors $\mathbf{b}_{k}^{H} \hat{=} p_{k} \mathbf{c}_{k}^{H}$ are defined, leading to the following simplified recursion:

$$
\mathbf{b}_{k}(l+1)=\mathbf{b}_{k}(l)+\mu E_{l}^{*}\left(1-E_{l} v_{k}^{*}(l)\right) \mathbf{r}_{k}(l)
$$

where $v_{k}(l) \hat{=} p_{k} u_{k}(l)=\mathbf{b}_{k}^{H} \mathbf{r}_{k}(l)$

\section{RESULTS}

In this section we show some results, with $N=31$ length spreading code, obtained in two different scenarios: i) a simulated channel with frequency selective fadings, multipath and white Gaussian noise; ii) a real ionospheric channel of about $800 \mathrm{Km}$ distance between Huelva and Barcelona in Spain (see [7] for more details). It is very important to remark that in the real link there are also other kind of corrupting signals such as narrow-band interferences, atmospheric noise, ionospheric irregularities, ... etc.

The proposed algorithms are compared with a standard RAKE receiver that uses the estimation of the power delay profile of the channel by crosscorrelating the received signal with the known pseudonoise sequence $\mathbf{p}$. After partitioning the module of the instantaneous crosscorrelation in N-block of data, a smooth average with a memory factor $\lambda_{R A K E}$ is applied. The maximum of the estimated power profile is detected and set to the central RAKE finger delay $\tau_{0}$. The $2 K+1$-finger RAKE uses all the information of the received signal included in a window delay between $\tau_{0}-K$ and $\tau_{0}+K$ The instantaneous cross-correlation samples inside this window are used to construct a decision variable that leads to the decoded information sequence.

\section{COMPUTER SIMULATIONS}

The simulated channel is a frequency selective multipath [1] and WSS channel [5] with an impulse response of the same length of the transmitted spreading code. Each $i$-tap coefficient is modeled as an independent slow time varying complex Gaussian process with zero-mean, a power $P(i)$ (which defines a Power delay profile shown in figure 6), a bandwidth (or Doppler spread) $B W_{D}=0.1 \mathrm{~Hz}$ and a Doppler shift $f_{D}=0.5$ $\mathrm{Hz}$. The only added interference is white complex Gaussian noise of power $\sigma^{2}$. The transmitted binary rate is $R_{s}=100$ bps.

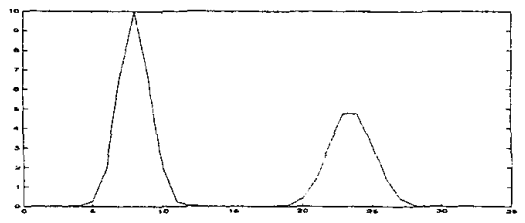

Fig. 6: Delay power profile of the simulated channel. Twohop propagation with a relative delay of about 4.8 msec. is here supposed. First path has almost twice the power of the second.

For the matrix-based $P N$ alignment filter the complete procedure of table 1 is carried out, using the variable step size parameter of equation (8), and the parameters are: $2 N=62$, $\delta=3 \cdot 10^{-6}, \alpha=10^{-9}$. On the other hand, equations of table 2 are used in the multi-filter $P N$ alignment equalizer, using the following values: $F=2, \mu=10^{-5}, \beta=10^{-3}$. RAKE receiver uses a memory factor $\lambda_{R A K E}=0.01$, and the number of used fingers is $2 K+1=5$, that allows to collect the energy of almost one path.

In table 3, the BER of the RAKE and the two proposed methods using the simulated channel is shown. As it is a time varying channel, the instantaneous SNR can achieve very low values, where unrecoverable errors occur.

\section{EVALUATION OF A REAL TRANSMISSION}

As it has been previously commented, some real transmissions through the real ionospheric link (see section II) have been carried out in order to validate the proposed method. Here only one single transmission is shown, but a better behavior of the new algorithms has been verified in the majority of the experiments. The transmitter power is set to $100 \mathrm{Watt}$, and the operating carrier frequency is $8.3 \mathrm{MHz}$. The binary data rate is $161 \mathrm{bps}$, and using a 31-chip Gold PN sequence, the transmitted DS-SS signal has a bandwidth of about $10 \mathrm{KHz}$.

The parameters of the blind equalizers are: $R=2 N=62$, $\delta=10^{-10}, \alpha=10^{-9}$ for the Blind matrix-based alignment 


\begin{tabular}{|c|c|c|c|}
\hline SNR $(\mathrm{dB})$ & BER $_{\text {RAKE }}$ & BER $_{\mathbf{1}}$ & BER $_{\mathbf{2}}$ \\
\hline-10 & 0.31 & 0.5 & 0.18 \\
\hline-5 & 0.18 & 0.49 & 0.0161 \\
\hline 0 & 0.14 & 0.044 & 0.0033 \\
\hline 5 & 0.012 & 0.0052 & 0.0039 \\
\hline 10 & 0.016 & 0.0063 & 0.0038 \\
\hline 15 & 0.018 & 0.0146 & 0.004 \\
\hline 20 & 0.0143 & 0.0137 & 0.0064 \\
\hline
\end{tabular}

Tab. 3: Results for different mean SNR in the simulated time varying multipath channel. Bit error probabilities of RAKE (BER RAKE), matrix-based filter algorithm (BER 1 ) and multichannel-based filter algorithm $\left(\mathbf{B E R}_{2}\right)$ are shown.

filter algorithm; and $F=2, \mu=10^{-8}, \beta=10^{-3}$ for the Blind multichannel alignment filter algorithm.

Next, some results of a single transmission of about $120 \mathrm{sec}$. are shown. In figure 7 are shown the equalized and detected sequence $E_{l}$ for both algorithms in function of time.

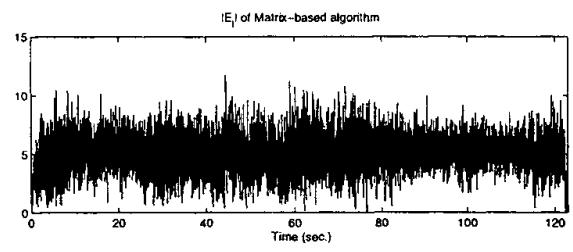

El a dinte (sec)

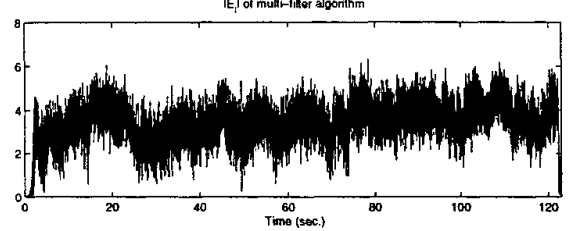

Fig. 7: Detected energy $E_{l}$ for the matrix-based algorithm (top) and the multichannel filter algorithm (bottom) in a real ionospheric transmission.

Error occurrence after the detection process is shown for the three demodulators in figure 8 . After the initial convergence period, errors occurrence is lower for the matrix-based algorithm if is compared with RAKE receiver. Finally, multichannel PN alignment filter gives the best performance in this experiment.

\section{CONCLUSIONS AND FUTURE WORK}

A new criterion for equalization and detection in a DS-SS system has been presented. Although the original formulation is based on the information of the transmitted spreading sequence, this can be omitted in the final solution. As can be seen, the final update and filtering procedure of equation (9) is almost the same as the well known LMS solution to CMA (Constant Modulus Algorithm) criterion [3]. This fact allow us to state that the presented optimization criterion in the matrix-based $P N$ alignment filter is equivalent to force the module of the output sequence to a constant value. Due to this fact, the second method (named multichannel-based PN alignment filter) can be interpreted as a certain multichannel CMA, as the optimizing criterion is exactly the same as the matrix-based PN alignment filter. Specifically, in equation (14) we can see a slight but interesting difference when $v_{k}^{*}(l)$ appears instead of $E_{l}^{*}$. Its computational simplicity and its better robustness in low SNR and time-variant multipath channels stand out from a RAKE receiver.

Regardless of the final simplifications, both methods have been conceived on the concept of $P N$ alignment. A unusual deterministic formulation of a cost function and a simple steepest ascent procedure have been used.

Future work will be done in order to extract channel estimation from the filter coefficients and to test its performance in multi-user communications.

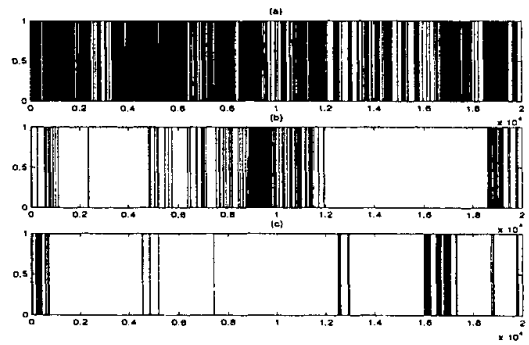

Fig. 8: Error occurrence ( 1 if there is an error and 0 if not) in function of the transmitted symbol of the RAKE receiver (a), the matrix-based filter (b) and the multichannel-based filter (c). Total bit error rates in this real ionospheric transmission are $0.15,0.021$ and 0.012 respectively.

\section{REFERENCES}

[1] J.G. Proakis Digital communications. Mc. Graw-Hill, New York, 1989.

[2] L. Tong, S. Perreau, "Multichannel blind identification: from subspace to maximum likelihood methods," Proceedings of the $I E E E$, vol. 86, pt. X, pp. October 1998.

[3] D.N. Godard, "Self-recovering equalization and carrier tracking in two-dimensional data communication systems", IEEE Tranactions on Communications, vol. 28, pp. 1867-1875, Nov. 1980

[4] J.C. Socoró, J.A. Moran, J.L. Pijoan, J.A. Montero, and C. Vilella, "Parameter estimation of wide-band channel model for digital communications system design", COST-251. Workshop on Ionosphere variability and channel characterization, Paris, September 1998.

[5] F. Arikan, O. Arikan : "Statistical characterization of time variability in midlatitude single tone $\mathrm{HF}$ channel response," 6th Management Committee and Working Group sessions of COST-251 Action, Linkping, Sweden, 8-11 October 1997

[6] J.A. Moran, J.L. Pijoan, J.R. Regué, C. Vilella, J.C. Socoró : "Sequential PN acquisition system based on fuzzy logic control," COST-262 4th Management Committee Meeting (MCM), 22-23 November 1999, Barcelona, Spain.

[7] J.L. Pijoan, J.R Regué, C. Vilella, J.A. Moran, J.C. Socoró : "DSP-based ionospheric radiolink using DS-SS," COST-262 4th Management Committee Meeting (MCM), 22-23 November 1999, Barcelona, Spain.

[8] J.Ll. Pijoan, J.C. Socoró, J.A. Moran, F.Tarres : "DSP-based ionospheric radiolink using DS-SS and on-line channel estimation," Proceedings of 10th Mediterranean Electrotechnical Conference (MELECON'2000), vol. II, pp. 860-863, 29-31 May, 2000 Limassol, Cyprus. 